\title{
Time evolution of ion fluxes incident at the substrate plane during reactive high-power impulse magnetron sputtering of groups IVb and VIb transition metals in Ar/N-2
}

Grzegorz Greczynski, Igor Zhirkov, Ivan Petrov, J oseph E Greene and J ohanna Rosén

The self-archived postprint version of this journal article is available at Linköping University Institutional Repository (DiVA):

http:// urn.kb.se/ resolve?urn=urn:nbn:se:liu:diva-147140

N.B.: When citing this work, cite the original publication.

Greczynski, G., Zhirkov, I., Petrov, I., Greene, J . E, Rosén, J ., (2018), Time evolution of ion fluxes incident at the substrate plane during reactive high-power impulse magnetron sputtering of groups $\mathrm{IVb}$ and VIb transition metals in Ar/ N-2, J ournal of Vacuum Science \& Technology. A. Vacuum, Surfaces, and Films, 36(2), 020602. https:// doi.org/ 10.1116/1.5016241

Original publication available at:

https:// doi.org/ 10.1116/ 1.5016241

Copyright: AIP Publishing

http:// www.aip.org/ 


\title{
Time evolution of ion fluxes incident at the substrate plane during reactive high-power impulse magnetron sputtering of Groups IVb and VIb transition metals in $\mathrm{Ar} / \mathrm{N}_{2}$
}

\author{
G. Greczynski, ${ }^{1}$ I. Zhirkov, ${ }^{1}$ I. Petrov, ${ }^{1,2}$ J.E. Greene, ${ }^{1,2,3}$ and J. Rosen ${ }^{1}$ \\ ${ }^{1}$ Department of Physics (IFM), Linköping University, SE-581 83 Linköping, Sweden \\ ${ }^{2}$ Materials Science Department and Frederick Seitz Materials Research Laboratory, University of \\ Illinois, Urbana, Illinois 61801 \\ ${ }^{3}$ Department of Physics, University of Illinois, Urbana, Illinois 61801, USA
}

\begin{abstract}
Reactive transition-metal (TM) nitride film growth employing bias-synchronized high power impulse magnetron sputtering (HiPIMS) requires a detailed knowledge of the time evolution of metal- and gas-ion fluxes incident at the substrate plane in order to precisely tune momentum transfer and, hence, provide the recoil density and energy necessary to eliminate film porosity at low deposition temperatures without introducing significant film stress. Here, we use energy- and time-dependent mass spectrometry to analyze the evolution of metal- and gas-ion fluxes at the substrate plane during reactive HiPIMS sputtering of Groups IVb and VIb TM targets in Ar/ $\mathrm{N}_{2}$ atmospheres. The time-and energy-integrated metal/gas ion ratio $N_{M e^{+}} / N_{g^{+}}$incident at the substrate is significantly lower for Group IVb TMs (ranging from 0.2 for Ti to 0.9 for Hf), due to high $\mathrm{N}_{2}$ reactivity which results in severely reduced target sputtering rates and, hence, decreased rarefaction. In contrast, for less reactive Group VIb metals, sputtering rates are similar to those in pure Ar as a result of significant gas heating and high $\mathrm{N}_{\mathrm{Me}^{+}} / \mathrm{N}_{g^{+}}$ratios, ranging from 2.3 for $\mathrm{Cr}$ to 98.1 for W. In both sets of experiments, the peak target current density is maintained constant at 1 $\mathrm{A} / \mathrm{cm}^{2}$. Within each TM group, $N_{M e^{+}} / N_{g^{+}}$scales with increasing metal-ion mass. For the GroupVIb elements, sputtered-atom Sigmund-Thompson energy distributions are preserved long after the HiPIMS pulse, in contradistinction to Group-IVb TMs for which the energy distributions collapse into narrow thermalized peaks. For all TMs, the $\mathrm{N}^{+}$flux dominates that of $\mathrm{N}_{2}{ }^{+}$ions, as the
\end{abstract}


molecular ions are collisionally dissociated at the target, and $\mathrm{N}^{+}$exhibits ion energy distribution functions resembling those of metal ions. The latter result implies that both $\mathrm{N}^{+}$and $\mathrm{Me}^{+}$species originate from the target. High-energy $\mathrm{Ar}^{+}$tails, assigned to ionized reflected-Ar neutrals, are observed with heavier TM targets.

corresponding author: grzgr@ifm.liu.se; phone: +4613281213 


\section{Introduction}

Metal-ion-synchronized high-power pulsed magnetron sputtering (HiPIMS), ${ }^{1,2}$ a technique developed for ion-assisted film growth, can provide additional control over layer composition and properties via significant ionization of the sputtered target atoms. ${ }^{3,4,5,6,7,8}$ Gas rarefaction, due to the presence of high temporal metal fluxes from the sputtering target, ${ }^{9,10,11,12}$ results in large variations in the intensities of gas- and metal-ion fluxes incident at the growing film surface during each HiPIMS pulse. ${ }^{13}$ Thus, by employing a substrate bias potential that is synchronized with the metal-ion-rich portion of the HiPIMS pulse, metal and gas ions incident at the substrate can be separated in both the time and energy domains. ${ }^{14,15}$ At low deposition temperatures, film nanostructure evolution is controlled by incident metal-ion energy and momentum transfer. The substrate is maintained at floating potential during the gas-ion portion of HiPIMS pulses; thus, the majority of gas ions arrive at the substrate with energies that are below the lattice displacement threshold. In contrast to substrate irradiation by fast gas-ions, conventionally employed in biased magnetically-unbalanced magnetron sputtering ${ }^{16,17}$ for which the gas ions are trapped in interstitial sites, metal-ions are primarily incorporated in lattice sites. This, together with dramatically-reduced concentrations of trapped gas ions, results in lower compressive stresses in layers deposited by metal-ion-synchronized HiPIMS.

The approach described above has been used to grow $\mathrm{N}$-doped $b c c-\mathrm{CrN}_{0.05}$ nanostructured films combining metallic and ceramic properties; ${ }^{18}$ hard, stress-free $\mathrm{Ti}_{0.39} \mathrm{Al}_{0.61} \mathrm{~N} ;{ }^{19}$ single-phase NaCl-structure $\mathrm{Ti}_{1-x} \mathrm{Si}_{x} \mathrm{~N}$ with extremely-high $\mathrm{SiN}$ concentrations; ${ }^{20}$ unprecedented AlN supersaturation in single-phase cubic $\mathrm{V}_{1-x} \mathrm{Al}_{x} \mathrm{~N} ;{ }^{21,22}$ and hard, dense $\mathrm{Ti}_{0.92} \mathrm{Ta}_{0.08} \mathrm{~N}$ and $\mathrm{Ti}_{0.41} \mathrm{Al}_{0.51} \mathrm{Ta}_{0.08} \mathrm{~N}$ alloys grown with no external heating. ${ }^{23,24}$ In each of these examples, the crucial step was synchronization of the substrate bias pulse to the metal-ion portion of the HiPIMS pulse, 
which requires detailed knowledge, typically provided by the time-resolved mass spectrometry, of the time evolution of metal- and gas-ion fluxes incident at the substrate.

Recently, we reported the results of energy- and time-dependent mass spectrometry analyses of species incident at the substrate plane during HiPIMS sputtering of Groups IVb and VIb transition metal (TM) targets in pure Ar. $^{25}$ We demonstrated that the time-and energyintegrated metal/gas ion ratio $N_{M e^{+}} / N_{A r^{+}}$increases with increasing peak target current density $J_{T, p e a k}$ as a result of gas rarefaction. Moreover, the effect scales with increasing metal-ion mass, resulting in $N_{M e^{+}} / N_{A r^{+}}$varying by two orders of magnitude from $\sim 1$ for Ti to $\sim 100$ for W with $J_{T, p e a k}=1 \mathrm{~A} / \mathrm{cm}^{2}$. We also showed that $N_{M e^{+}} / N_{A r^{+}}$can be controlled over a wide range by

adjusting the HiPIMS pulse width $\tau_{O N .}{ }^{26}$ For Ti sputtered in $\operatorname{Ar}\left(m_{M e} / m_{g}=1.2\right), N_{T i^{+}} / N_{A r^{+}}$varies from $\sim 1$ with $\tau_{\mathrm{ON}}=120 \mu$ s to $\sim 60$ for $\tau_{\mathrm{ON}}=30 \mu \mathrm{s}$. Thus, shortening the HiPIMS pulse, provides more flexibility in choosing bias pulse shapes, independent of the choice of metal/gas combination.

Here, we report the results of a subsequent study in which we investigate the evolution of metal- and gas-ion fluxes incident at the substrate plane during reactive HiPIMS of Groups IVb and VIb TM targets in Ar/ $\mathrm{N}_{2}$ gas mixtures. We show that the time- and energy-integrated metal/gas-ion ratios $N_{M e^{+}} / N_{A r^{+}}$incident at the substrate plane are higher for Group VIb metals due to lower $\mathrm{N}_{2}$ reactivity, resulting in lower target coverages, higher target sputtering rates, and, hence, less gas rarefaction. Ion energy distribution functions for Zr, Hf, Mo, and W targets operated in reactive HiPIMS mode are reported for the first time.

\section{Experimental procedure}

Time-dependent in-situ mass- and energy-spectroscopy analyses of ion fluxes during HiPIMS sputtering of Ti, Zr, Hf, Cr, Mo, and W targets (99.99 \% pure) in $\mathrm{Ar} / \mathrm{N}_{2}$ gas mixtures are performed 
using a Hiden Analytical EQP1000 instrument. Experiments are carried out in a CemeCon CC800/9 magnetron sputtering system equipped with rectangular $8.8 \times 50 \mathrm{~cm}^{2}$ targets parallel to, and $18 \mathrm{~cm}$ from, the electrically-grounded orifice of a mass spectrometer placed at the normal substrate position. The system base pressure is $2 \times 10^{-4} \mathrm{~Pa}\left(1.5 \times 10^{-6}\right.$ Torr $)$, and the sputtering pressure is maintained constant at $P=0.4 \mathrm{~Pa}$ (3 mTorr), with a $\mathrm{N}_{2} / \mathrm{Ar}$ flow ratio of 0.11 , which yields stoichiometric Group IVb TM nitride films as determined by Rutherford backscattering spectrometry and time-of-flight elastic recoil detection analysis.

The HiPIMS pulse length is $120 \mu$ s at a frequency of $300 \mathrm{~Hz}$. In order to maintain the same peak target current density $J_{\text {T,peak }}=1.0 \mathrm{~A} / \mathrm{cm}^{2}$, the average HiPIMS power $P_{\text {avg }}$ ranges from 1500 W for Zr to 1900, 2400, 3000, 3200, and $4000 \mathrm{~W}$ for Ti, Hf, Mo, Cr, and W target, respectively. Ion-energy distribution functions (IEDFs) $I_{M e^{n+}}\left(E_{i}\right)$ are recorded in HiPIMS mode for $\mathrm{Me}^{n+}(n=$ $1,2, \ldots)$ and gas ions during 100 consecutive pulses such that the total acquisition time per data point is $1 \mathrm{~ms}$. The ion energy is scanned in $0.5 \mathrm{eV}$ steps from $E_{i}=1$ to $50 \mathrm{eV}$. To obtain the plasma composition at the substrate plane, where the orifice of the mass spectrometer is positioned, rather than at the detector, data are corrected for the ion time-of-flight (TOF) through the instrument using the approach described in Ref. 27. Additional details regarding the IEDF measurements are given in Ref. 28.

\section{Results and Discussion}

Target voltage waveforms $V_{T}(t)$ recorded during reactive sputtering of Groups IVb (Ti, Zr, and $\mathrm{Hf}$ ) and VIb (Cr, Mo, and W) targets in Ar/ $\mathrm{N}_{2}$ atmospheres with a peak target current density of $1 \mathrm{~A} / \mathrm{cm}^{2}$ are shown in Fig. 1 . For all targets, negative $V_{T}(t)$ values decrease with time throughout each pulse as the discharge becomes power-supply limited. The starting ( $t=0 \mu \mathrm{s})$ and end ( $t=120$ 
$\mu$ s) $V_{T}$ values are lower for Group IVb TM targets: -600 to $-250 \mathrm{~V}$ for $\mathrm{Ti}$, -510 to $-180 \mathrm{~V}$ for $\mathrm{Zr}$, and -660 to $265 \mathrm{~V}$ for Hf. With Group VIb targets, $V_{T}$ ranges from -795 to $-390 \mathrm{~V}$ for $\mathrm{Cr},-760$ to $-320 \mathrm{~V}$ for Mo, and -970 to $-490 \mathrm{~V}$ for $\mathrm{W}$.

Fig. 2 shows time-dependent intensities of energy-integrated primary-ion fluxes $\mathrm{Me}^{+}, \mathrm{Me}^{2+}$, $\mathrm{Ar}^{+}, \mathrm{N}_{2}{ }^{+}$, and $\mathrm{N}^{+}$, recorded while reactively sputtering Groups IVb and VIb TM targets in $\mathrm{Ar} / \mathrm{N}_{2}$ atmospheres with a peak target current density of $1 \mathrm{~A} / \mathrm{cm}^{2}$. Results are plotted with a $10 \mu \mathrm{s}$ resolution. Zero on the time axis corresponds to the onset of the cathode voltage pulse; each data point at time $t$ represents the number of ions collected during the interval from $t-5$ to $t+5 \mu$ s. For both IVb and VIb targets, the gas-ion flux $F_{g^{+}}(t)$ (in which $g^{+}$includes $\mathrm{Ar}^{+}, \mathrm{N}_{2}{ }^{+}$, and $\mathrm{N}^{+}$) decreases with increasing metal-ion mass. In the case of IVb metals, the energy and time-integrated metal/gas ion ratio $N_{M e^{+}} / N_{g^{+}}$, defined as $\int F_{M e^{+}}(t) d t / \int F_{g^{+}}(t) d t$ (in which $F_{M e^{+}}(t)$ is the metal-ion flux) with the integral extending between $t=0$ and $300 \mu$ s, increases from 0.2 for $\operatorname{Ti}\left(m_{T i}=47.87 \mathrm{amu}\right)$, to 0.7 for $\mathrm{Zr}\left(m_{Z r}=91.22 \mathrm{amu}\right)$, and 0.9 for $\mathrm{Hf}\left(m_{H f}=178.49 \mathrm{amu}\right)$. In a similar manner for VIb targets, $N_{\mathrm{Me}^{+}} / N_{\mathrm{Ar}^{+}}=2.3$ for $\mathrm{Cr}\left(m_{\mathrm{Cr}}=52.00 \mathrm{amu}\right)$, and increases to 36.6 and 98.1 for $\mathrm{Mo}\left(m_{\mathrm{Mo}}=\right.$ $95.94 \mathrm{amu})$ and $\mathrm{W}\left(m_{W}=183.84 \mathrm{amu}\right)$, respectively. The increase in $N_{M e^{+}} / N_{g^{+}}$with increasing TM mass, previously reported for non-reactive HiPIMS, ${ }^{25}$ is caused by gas rarefaction effects, which scale with increasing metal/gas atom mass ratio $m_{M e} / m_{g}$. That is, the metal/gas collision cross-section increases with increasing sputtered atom mass, resulting in shorter metal-atom mean free paths, increased momentum transfer, and hence more effective gas heating. The control experiments performed for selected targets as a function of $P_{\text {avg }}$ at constant frequency (corresponding to varying $J_{T, p e a k}$ over a wide range) revealed that the variation in $N_{\mathrm{Me}^{+}} / N_{\mathrm{g}^{+}}$ratio with $P_{\text {avg }}$ is minor as compared to that observed between different targets. 
Another important effect illustrated in Fig. 2 is that gas-ion fluxes are significantly lower for VIb than IVb metal targets (comparing elements with similar masses). This effect is caused by higher temporal fluxes of sputter-ejected species from Group VIb targets due to both (i) higher sputter yields $S_{A r \rightarrow M e}$ and $S_{M e \rightarrow M e}$, and (ii) lower reactivity towards nitrogen (see Table 1), which leads to a lower rate of compound formation at the target surface. The latter is also evident from the reduction in $\mathrm{N}_{\mathrm{Me}^{+}} / N_{\mathrm{g}^{+}}$, which is more severe for IVb than for VIb TM targets, when comparing metallic ${ }^{25}$ to reactive sputtering. For example, with a Hf target, which has a relatively high heat of nitride formation, $\Delta H_{f}^{0}=-3.7 \mathrm{eV} /$ atom, $N_{M e^{+}} / N_{g^{+}}$decreases by a factor of 33 upon adding $\mathrm{N}_{2}$ to Ar, while the corresponding drop in $N_{\mathrm{Me}^{+}} / N_{g^{+}}$is only $11 \%$ for a W target with $\Delta H_{f}^{0}$ $=-0.2 \mathrm{eV} /$ atom. The increase in $N_{\mathrm{Me}^{+}} / N_{g^{+}}$with increasing TM mass, is more pronounced for VIb metals due to the simultaneous decrease in $\mathrm{N}_{2}$ reactivity, unlike the case for Group IVb TMs for which $\Delta H_{f}^{0}$ remains approximately constant between -3.4 and -3. 7 eV/atom (see Table 1).

A direct consequence of the above phenomena is that the time-separation of metal- and gasion fluxes at the substrate, critical for synchronized-bias HiPIMS film growth, is much more difficult to achieve during reactive sputtering of Group IVb targets, for which $\mathrm{Ar}^{+}$and $\mathrm{N}^{+}$gas-ion fluxes are significantly larger than those obtained using Group VIb targets. The intense atomicnitrogen ion fluxes $F_{N^{+}}(t)$, which dominate the molecular $\mathrm{N}_{2}{ }^{+}$ion fluxes in agreement with previous reports, ${ }^{15}$ peak at 50-60 $\mu$ s from pulse initiation and precede the metal ions by 15-50 $\mu$ s (depending on $m_{M e}$ ) due to shorter flight times from the target. However, $\mathrm{N}^{+}$is a film-forming ion, hence the overlap with metal-ion fluxes is not an issue. $F_{A r^{+}}(t)$ fluxes, however, are far more problematic; they can result in trapped Ar interstitials leading to higher residual stress ${ }^{29,30}$ and, as a consequence, to cohesive and/or adhesive film failure. ${ }^{31,32} \mathrm{~A}$ possible remedy is the use of shorter HiPIMS pulses, while maintaining the peak target current density constant. In the case of metallic 
operation, this approach results in a significant increase in the $N_{M e^{+}} / N_{A r^{+}}$ratio. ${ }^{26}$ For example, by shortening the HiPIMS pulse in reactive mode from 120 to $30 \mu$ s while sputtering Ti, $N_{T^{+}} / N_{A r^{+}}$ at the substrate plane increases from 0.2 to 0.5 (not shown). In contrast, during reactive sputtering of Group VIb targets, for which metal-ions dominate the ionized flux incident at the substrate, the bias length and offset can be set based upon measured $F_{\mathrm{Me}^{+}}(t)$ distributions.

Another important observation for metal-ion-synchronized reactive HiPIMS is that the time delays between the maxima in target current and subsequent $F_{\mathrm{Me}^{+}}(t)$ peaks, reflecting the ion flight time from the target to the substrate plane, $\tau_{T O F}$, increase with increasing metal-ion mass, independent of the target current density. This is shown in Fig. 3 for Ti, Zr, Hf, Cr, Mo, and W, all plotted as a function of peak target current density $J_{T, p e a k}$. Data for Al $\left(m_{A l}=26.98 \mathrm{amu}\right)$ are included to further illustrate the effect of ion mass on $\tau_{T O F}$. In the limit of low peak target current density, $J_{T, p e a k}=0.1 \mathrm{~A} / \mathrm{cm}^{2}, \tau_{T O F}$ varies from $41 \mu$ s for Al, to $60 \pm 2 \mu$ s for Ti and $\mathrm{Cr}$, to $71 \pm 1 \mu$ s for $\mathrm{Zr}$ and Mo, and $92 \mu$ s for both Hf and W. The decrease in $\tau_{T O F}$ with increasing $J_{T, p e a k}$, which occurs for all metal ions investigated, stems primarily from the reduced number of collisions with gas particles as rarefaction increases, as well as from the increase in the average metal sputter-ejection energy. ${ }^{33,34}$ At the opposite extreme, $J_{T, p e a k}=1 \mathrm{~A} / \mathrm{cm}^{2}, \tau_{T O F}$ varies from $18 \mu$ s for Al, to $34 \pm 1 \mu \mathrm{s}$ for Ti and $\mathrm{Cr}$, to $47 \pm 1 \mu$ s for $\mathrm{Zr}$ and Mo, to $61 \pm 1 \mu$ s for $\mathrm{Hf}$ and $\mathrm{W}$. Hence, in order to fully utilize metal-ion bombardment in the reactive mode, the duration of synchronous substrate-bias pulses should be increased with increasing metal-ion mass. Since the metal-ion energy distribution varies over a wide range (see discussion below), one consequence is that metal-ion peaks are broader for heavier ions, except for the relatively light mass $\mathrm{Ti}^{+}$for which $F_{T i^{+}}(t)$ exhibits a slowly-decaying tail long after the $120 \mu$ s HiPIMS pulse. 
Fig. 4 presents $\mathrm{Me}^{+}, \mathrm{Me}^{2+}, \mathrm{Ar}^{+}, \mathrm{N}_{2}^{+}$, and $\mathrm{N}^{+}$IEDFs at the substrate plane during 300- $\mu$ s-long time intervals, starting with the ignition of $120-\mu$ s HiPIMS pulses, while sputtering TM targets in $\mathrm{Ar} / \mathrm{N}_{2}$ at a peak current density of $1 \mathrm{~A} / \mathrm{cm}^{2}$. Only the $\mathrm{Ti}^{+}$IEDF exhibits a narrow low-energy peak at 2-3 eV (representing the difference between the bulk plasma potential and the grounded orifice), as a result of thermalization after the HiPIMS pulse $(t>150 \mu \mathrm{s})$. That is, the sputtered Ti (47.87 amu) species lose significant energy via scattering collisions with Ar (39.95 amu) atoms, as the target current density and, hence, degree of rarefaction decreases (i.e., the local Ar pressure increases). ${ }^{35}$ All other metal IEDFs exhibit broad Sigmund-Thompson-type sputtered-species energy distributions; ${ }^{36,37}$ indicating that the sputter-ejected atoms undergo few collisions between the target and substrate plane due to efficient plasma rarefaction. ${ }^{38}$ The effect is particularly pronounced for $\mathrm{W}(183.84 \mathrm{amu})$, for which IEDFs extend out to $100 \mathrm{eV}$. This is consistent with $\mathrm{W}^{+}$ions having the highest $N_{\mathrm{Me}^{+}} / N_{g^{+}}$ratio, indicative of strong rarefaction due to a high atomic mass and a low heat of nitride formation (see Table 1).

Gas-ion IEDFs at the substrate plane display significant differences when reactively sputtering Group IVb vs. VIb TM targets. As depicted in Fig. 4, in the former case both $\mathrm{Ar}^{+}$and $\mathrm{N}_{2}{ }^{+}$IEDFs have strong thermalized peaks, which are absent during reactive sputtering of Group VIb TMs. This is a direct consequence of differences in the temporal fluxes of sputter-ejected species, which are significantly higher for Group VIb targets, characterized by higher sputter yields and lower reactivities toward $\mathrm{N}_{2}$, both of which exacerbate gas rarefaction. For all TM targets investigated, $I_{N^{+}}\left(E_{i}\right)$ closely resemble the corresponding $\mathrm{Me}^{+}$IEDFs. This is strong evidence that $\mathrm{N}^{+}$ions originate from the target, in agreement with previous reports. ${ }^{28}$ The source of $\mathrm{N}^{+}$is a combination of sputter-ejected $\mathrm{N}$ atoms and reflected $\mathrm{N}$ atoms arising from dissociative $\mathrm{N}_{2}{ }^{+}$ collisions at the target surface. 
With increasing TM mass, $I_{A r^{+}}\left(E_{i}\right)$ IEDFs develop high-energy tails (see Fig. 4). For the lighter TM elements, Ti and $\mathrm{Cr}, I_{A r^{+}}\left(E_{i}\right)$ decreases below $10^{3}$ cps at $E_{i} \gtrsim 20 \mathrm{eV}$. However, $I_{A r^{+}}\left(E_{i}\right)$ remains above $10^{3}$ cps until $E_{i} \simeq 40$ and $45 \mathrm{eV}$, while sputtering $\mathrm{Zr}$ and W targets, respectively. For Mo and Hf, $I_{A r^{+}}\left(E_{i}\right)$ exceeds $10^{3}$ cps even at $E_{i}=50 \mathrm{eV}$. The most extreme case is that of $\mathrm{Hf}$, for which $I_{A r^{+}}\left(E_{i}\right)$ is still $10^{4}$ cps with $E_{i}=80 \mathrm{eV}$. Since the effect scales with increasing $m_{M e}$, we assign it to $\mathrm{Ar}^{+}$ions neutralized at the target, reflected toward the substrate plane, and re-ionized while passing through the dense plasma region. ${ }^{39,40,41}$ These high-energy species are typically observed when $m_{g}<<m_{M e}$, for which there is a high probability of $\mathrm{Ar}^{+}$ions being reflected with a significant fraction of the incident energy. ${ }^{42,43}$ Interestingly, high-energy tails are never observed in $I_{N_{2}^{+}}\left(E_{i}\right)$ IEDFs for any TM target investigated. $I_{A r^{+}}\left(E_{i}\right)$ and $I_{N_{2}^{+}}\left(E_{i}\right)$ are similar for $0 \leq E_{i} \lesssim 15$ $\mathrm{eV}$, but differ significantly at higher ion energies. This can be explained by $\mathrm{N}_{2}{ }^{+}$dissociation upon target impact resulting in a high probability of atomic $\mathrm{N}$ reflection since $m_{N}<<m_{M e}$.

Additional insight into the temporal evolution of $\mathrm{Ar}^{+}$fluxes at the substrate plane derives from the time-resolved $I_{A r^{+}}\left(E_{i}\right)$ IEDFs shown in Fig. 5. The spectra are recorded during consecutive 20- $\mu$ s time intervals throughout, and following, $120-\mu$ s HiPIMS pulses in $\mathrm{Ar} / \mathrm{N}_{2}$ gas mixtures. As noted earlier, there is a very pronounced difference in the results for Group IVb vs. VIb reactive sputtering. In the former case, the original $I_{A r^{+}}\left(E_{i}\right)$ IEDFs with broad energy distributions observed at $30 \lesssim t \lesssim 90 \mu$ s collapse into narrow thermalized peaks at $t \gtrsim 90 \mu$ s. In contrast, for VIb TM targets, with the exception of $\mathrm{Cr}$ which shows some indication of thermalization, $I_{A r^{+}}\left(E_{i}\right)$ IEDFs are preserved throughout the entire measurement period up to 250 $\mu$ s. That is, they do not collapse into low-energy thermalized peaks, as observed for IVb metal targets. Instead, there is a gradual loss in intensity and even the ions arriving after the HiPIMS pulse, $t \geq 120 \mu \mathrm{s}$, have an average energy that is significantly higher than that of thermalized ions. 
The dramatic decrease in thermalized ion density, despite the fact that the thermalization length $\lambda_{\text {th }}$ (see Table 1) ${ }^{44}$ corresponding to the distance at which the initial ion velocity is reduced to thermal velocity, is significantly shorter than the target-to-orifice distance, indicates severe rarefaction and is consistent with the fact that the energy and time-integrated metal/gas-ion ratio $N_{\mathrm{Me}^{+}} / \mathrm{N}_{\mathrm{Ar}^{+}}$is higher for VIb TMs.

\section{Conclusions}

In summary, we performed energy- and time-dependent mass spectrometry analyses of metal- and gas-ion fluxes incident at the substrate plane during HiPIMS reactive sputtering of Groups IVb and VIb TM targets in $\mathrm{Ar} / \mathrm{N}_{2}$ atmospheres. Unlike the case for sputtering in pure $\mathrm{Ar}$, for which gas rarefaction effects scale primarily with the metal/gas mass ratio $m_{\mathrm{Me}} / \mathrm{m}_{g}$, target reactivity with nitrogen, as quantified by the heat of nitride formation $\Delta H_{f}^{0}$, plays a dominant role as compound formation at the target surface dramatically reduces sputtering rates and, hence, gas heating. Thus, time-and energy-integrated metal/gas-ion ratios $N_{\mathrm{Me}^{+}} / N_{g^{+}}$at the substrate plane, which are controlled by gas rarefaction, are significantly lower for Group IVb TM targets. This, in turn, means that during reactive sputtering of Group IVb TMs, the metal-ion flux distributions $F_{M e^{+}}(t)$ significantly overlap the gas-ion flux $F_{g^{+}}(t)$ distributions, thus inhibiting the selective manipulation of metal-ion energy and momentum via synchronized biasing. A potential remedy is the use of shorter HiPIMS pulses which result in higher $\mathrm{N}_{\mathrm{Me}^{+}} / N_{\mathrm{g}^{+}}$values and decreased $F_{\mathrm{Me}}(t)$ overlap with $F_{g^{+}}(t)$. In contrast, with less reactive VIb TM targets, sputtering rates are similar to those in pure $\operatorname{Ar}$ (i.e., closer to the metallic mode), leading to very high $N_{\mathrm{Me}^{+}} / N_{g^{+}}$ratios. For all Group VIb TM targets, metal-ions dominate the ionized flux to the substrate and the bias length and offset can be set based upon the measured $F_{\mathrm{Me}^{+}}(t)$ distribution functions. 


\section{Acknowledgements}

Financial support from the Swedish Research Council VR Grant 2014-5790, an Åforsk

foundation grant \#16-359, the Swedish Government Strategic Research Area in Materials Science on Functional Materials at Linköping University (Faculty Grant SFO Mat LiU No. 2009 00971), and the Knut and Alice Wallenberg foundation Fellowship Grant and Project funding (KAW 2015.0043) is gratefully acknowledged. 


\section{Figure captions}

Fig. 1. (Color online) Target voltage $V_{T}(t)$ waveforms recorded during reactive HiPIMS sputtering of Groups IVb (Ti, Zr, and Hf) and VIb (Cr, Mo, and W) TM targets in $0.4 \mathrm{~Pa} \mathrm{(3} \mathrm{mTorr)}$ $\mathrm{Ar} / \mathrm{N}_{2}$ atmospheres at a peak target current density of $1 \mathrm{~A} / \mathrm{cm}^{2}$.

Fig. 2. (Color Online) Time evolution of the energy-integrated $\mathrm{Me}^{+}, \mathrm{Me}^{2+}, \mathrm{Ar}^{+}, \mathrm{N}_{2}{ }^{+}$, and $\mathrm{N}^{+}$ion fluxes $F(t)$, incident at the substrate plane during reactive HiPIMS sputtering of Groups IVb (Ti, Zr, and Hf) and VIb (Cr, Mo, and W) TM targets in $0.4 \mathrm{~Pa} \mathrm{(3} \mathrm{mTorr)} \mathrm{Ar} / \mathrm{N}_{2}$ atmospheres at a peak target current density of $1 \mathrm{~A} / \mathrm{cm}^{2}$. Grey dashed curves are target current pulses $J_{T}(t)$ scaled to match the $F_{M e^{+}}(t)$ intensities in order to facilitate comparison.

Fig. 3. (Color Online) Metal ion times-of-flight $\tau_{T O F}$ from the target to the substrate plane plotted as a function of peak target current density $J_{T, p e a k}$ during reactive HiPIMS sputtering of metal targets (Al, Ti, Zr, Hf, Cr, Mo, and $\mathrm{W}$ ) in $\mathrm{Ar}$ at $0.4 \mathrm{~Pa}$ (3 mTorr). $\tau_{\text {TOF }}$ values are extracted from the time difference between $F_{M e^{+}}(t)$ and $J_{T}(t)$ maxima. Numbers in parentheses indicate metal ion mass expressed in atomic mass units.

Fig. 4. (Color Online) $\mathrm{Me}^{+}, \mathrm{Me}^{2+}, \mathrm{Ar}^{+}, \mathrm{N}_{2}{ }^{+}$, and $\mathrm{N}^{+}$ion energy distribution functions (IEDFs) recorded at the substrate position during reactive HiPIMS sputtering of Ti, Zr, Hf, Cr, Mo, and W targets in $0.4 \mathrm{~Pa}$ (3 mTorr) $\mathrm{Ar} / \mathrm{N}_{2}$ atmospheres at a peak target current density of 1 $\mathrm{A} / \mathrm{cm}^{2}$. The IEDFs are acquired during 300- $\mu$ s-long time intervals starting from pulse ignition. The HiPIMS pulse length is $120 \mu$ s. 
Fig. 5. (Color Online) $\mathrm{Ar}^{+}$ion energy distribution functions (IEDFs) recorded at the substrate position during reactive HiPIMS sputtering of $\mathrm{Ti}$, Zr, Hf, Cr, Mo, and W targets in $0.4 \mathrm{~Pa}$ (3 mTorr) Ar/ $\mathrm{N}_{2}$ atmospheres at a peak target current density of $1 \mathrm{~A} / \mathrm{cm}^{2}$. The IEDFs are acquired during $20-\mu$ s time intervals over the time period from 0 (pulse ignition) to $250 \mu \mathrm{s}$. The pulse length is $120 \mu \mathrm{s}$.

\section{Table caption}

Table 1. Relevant parameters for analyzing ion fluxes incident at the substrate plane during reactive HiPIMS of Groups IVb and VIb TM targets in Ar/ $\mathrm{N}_{2}$ gas mixtures. TM mass $m_{M e},{ }^{45}$ first and second metal ionization potentials $I P_{M e}^{1}$ and $I P_{M e}^{2},{ }^{46} \mathrm{TRIM}^{47}$ Ar sputtering and metal self-sputtering yields $S_{A r \rightarrow M e}$ and $S_{M e \rightarrow M e}$ for incident ion energies (in parentheses) corresponding to the target voltage at $J_{T, p e a k}$, the heat of nitride formation $\Delta H_{f}^{0},{ }^{48}$ and the thermalization distance $\lambda_{\text {th }}$ for TM atoms sputter-ejected with $10 \mathrm{eV}$ in the forward direction. $^{25}$ 


\section{References}

${ }^{1}$ V. Kouznetsov, K. Macak, J. M. Schneider, U. Helmersson and I. Petrov, Surf. Coat. Technol. 122, 290 (1999).

${ }^{2}$ K. Sarakinos, J. Alami, S. Konstantinidis, Surf. Coat. Technol. 204, 1661 (2010).

${ }^{3}$ J. Alami, K. Sarakinos, G. Mark, M. Wuttig, Appl. Phys. Lett. 89, 154104 (2006).

${ }^{4}$ M. Desecures, L. de Poucques, T. Easwarakhanthan, and J. Bougdira, Appl. Phys. Lett. 105, 181120 (2014).

${ }^{5}$ S. Loquai, O. Zabeida, J. E. Klemberg-Sapieha, and L. Martinu, Appl. Phys. Lett. 109, 114101 (2016).

${ }^{6}$ A. Hecimovic, N. Britun, S. Konstantinidis, and R. Snyders, Appl. Phys. Lett. 110, 014103 (2017).

${ }^{7}$ P. Raman, J. Weberski, M. Cheng, I. Shchelkanov, and D. N. Ruzic, J. Appl. Phys. 120, 163301 (2016).

${ }^{8}$ G. Greczynski, I. Petrov, J.E. Greene, and L. Hultman, Vacuum 116, 36 (2015).

${ }^{9}$ U. Helmersson, M. Lattemann, J. Bohlmark, A. P. Ehiasarian, and J. T. Gudmundsson, Thin Solid Films 513, 1 (2006).

${ }^{10}$ D. Horwat, A. Anders, J. Appl. Phys. 108, 123306 (2010).

${ }^{11}$ A. Anders, Surf. Coat. Technol. 205, S1 (2011).

${ }^{12}$ N. Britun, S. Konstantinidis, R. Snyders, Plasma Processes and Polymers 12, 1010 (2015).

${ }^{13}$ K. Macak, V. Kouznetsov, J. Schneider, U. Helmersson, I. Petrov, J. Vac. Sci. Technol. A 18, 1533 (2000).

${ }^{14}$ G. Greczynski et al., J. Vac. Sci. Technol. A 30, 061504 (2012).

${ }^{15}$ G. Greczynski et al., Surf. Coat. Technol. 257, 15 (2014).

${ }^{16}$ I. Petrov, L. Hultman, J.-E. Sundgren, and J. E. Greene, J. Vac. Sci. Technol. A 10, 265 (1992).

${ }^{17}$ I. Petrov, P.B. Barna, L. Hultman, J.E. Greene, J. Vac. Sci. Technol. A 21, 117 (2003).

${ }^{18}$ G. Greczynski, J. Lu, O. Tengstrand, I. Petrov, J.E. Greene, and L. Hultman, Scripta Mat. 122, 40 (2016).

${ }^{19}$ G. Greczynski et al., Thin Solid Films 556, 87 (2014).

${ }^{20}$ G. Greczynski et al., Surf. Coat. Technol. 280, 174 (2015).

${ }^{21}$ G. Greczynski et al., J. Appl. Phys. 121, 171907 (2017).

${ }^{22}$ G. Greczynski, S. Mráz, H. Ruess, M. Hans, J. Lu, L. Hultman, J.M. Schneider, J. Appl. Phys. 122, 025304 (2017).

${ }^{23}$ G. Greczynski et al., J. Vac. Sci. Technol. A 32, 041515 (2014).

${ }^{24}$ H. Fager et al., J. Appl. Phys. 121, 171902 (2017).

${ }^{25}$ G. Greczynski, I. Zhirkov, I. Petrov, J.E. Greene, and J. Rosen, J. Vac. Sci. Technol. A 35, 060601 (2017).

${ }^{26}$ G. Greczynski, I. Zhirkov, I. Petrov, J.E. Greene, and J. Rosen, Thin Solid Films 642, 36 (2017).

27 J. Bohlmark, M. Lattemann, J.T. Gudmundsson, A.P. Ehiasarian, Y.A. Gonzalvo, N. Brenning, U. Helmersson, Thin Solid Films 515, 1522 (2006).

${ }^{28}$ G. Greczynski and L. Hultman, Vacuum 84, 1159 (2010).

${ }^{29}$ C.A. Davis, Thin Solid Films 226, 30 (1993).

${ }^{30}$ S. Ulrich, T. Theel, J. Schwan, H. Ehrhardt, Surf. Coat. Technol. 97, 45 (1997).

${ }^{31}$ V. Teixeira, Thin Solid Films 392, 276 (2001).

${ }^{32}$ H. Oettel, and R. Wiedemann, Surf. Coat. Technol. 76, 265 (1995).

${ }^{33}$ M. Panjan, R. Franz, and A. Anders, Plasma Sources Sci. Technol. 23, 025007 (2014).

${ }^{34}$ R. Franz, C. Clavero, J. Kolbeck, and A. Anders, Plasma Sources Sci. Technol. 25, 015022 (2016).

${ }^{35}$ The large difference between $\mathrm{Ti}^{+}$and $\mathrm{Cr}^{+}$IEDFs in Figure 4 is due to a correspondingly large difference in sputterejected metal fluxes from the two targets. The sputter yield for Ti, low in metallic mode (see Table 1), becomes further reduced in reactive mode due to target poisoning caused by high reactivity with $\mathrm{N}_{2}$ (compared to $\mathrm{Cr}$ ).

${ }^{36}$ P. Sigmund, J. Vac. Sci. Technol. A 17, 396 (1979).

${ }^{37}$ M. W. Thompson, Physics Reports 69, 335 (1981).

38 J.T. Gudmundsson, N. Brenning, D. Lundin, and U. Helmersson, J. Vac. Sci. Technol. A, 30030801 (2012).

${ }^{39}$ D.W. Hoffman and J.A. Thornton, J. Vac. Sci. Technol. 17, 380 (1980).

${ }^{40}$ M. Misina, L.R. Shaginyan, M. Macek, and P. Panjan, Surf. Coat. Technol. 142-144, 348 (2001).

${ }^{41}$ J.A. Thornton, J. Tabock and D.W. Hoffman, Thin Solid Films 64, 111 (1979).

${ }^{42}$ B. Window, Surf. Coat. Technol. 71, 93 (1995).

${ }^{43}$ The much longer extended high-energy tail in $\mathrm{Ar}^{+}$IEDFs (see Figure 4) recorded during HiPIMS sputtering of Hf compared to $\mathrm{W}$, which have similar atomic masses (both $~ 4.4$ times that of Ar), is another consequence of $\mathrm{Ar}$ rarefaction. The degree of rarefaction is considerably higher for W HiPIMS due to higher $\mathrm{Ar}^{+}$and self-sputtering yields together with a lower reactivity with $\mathrm{N}_{2}$ (see Table 1 ). The latter results in decreased target poisoning and, hence, much higher reactive sputtering rates. 
${ }^{44}$ W.D. Westwood, J. Vac. Sci. Technol. 15, 1 (1978).

45 J.C. Slater, J. Chem. Phys. 41, 3199 (1964).

${ }^{46}$ David R. Lide (ed), CRC Handbook of Chemistry and Physics, 84th Edition. CRC Press. Boca Raton, Florida, 2003; Section 10, Atomic, Molecular, and Optical Physics; Ionization Potentials of Atoms and Atomic Ions.

${ }^{47}$ J. F. Ziegler, J. P, Biersack, U. Littmark, "The Stopping and Range of Ions in Solids", Pergamon Press, New York (1984)

${ }^{48}$ M.W. Chase, Jr., NIST-JANAF Thermochemical Tables, Fourth Edition, J. Phys. Chem. Ref. Data, Monograph 9 (1998) 1-1951 


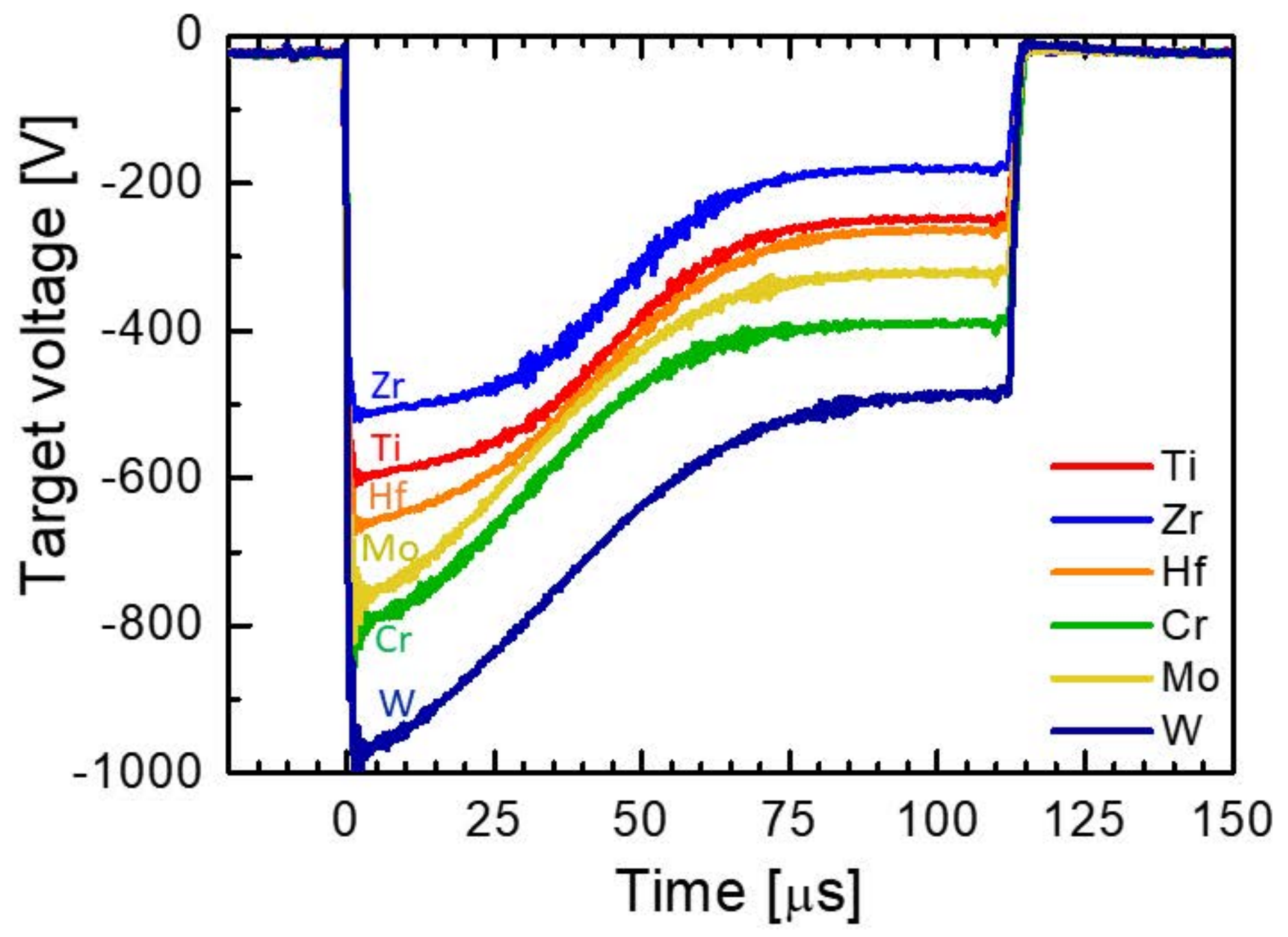



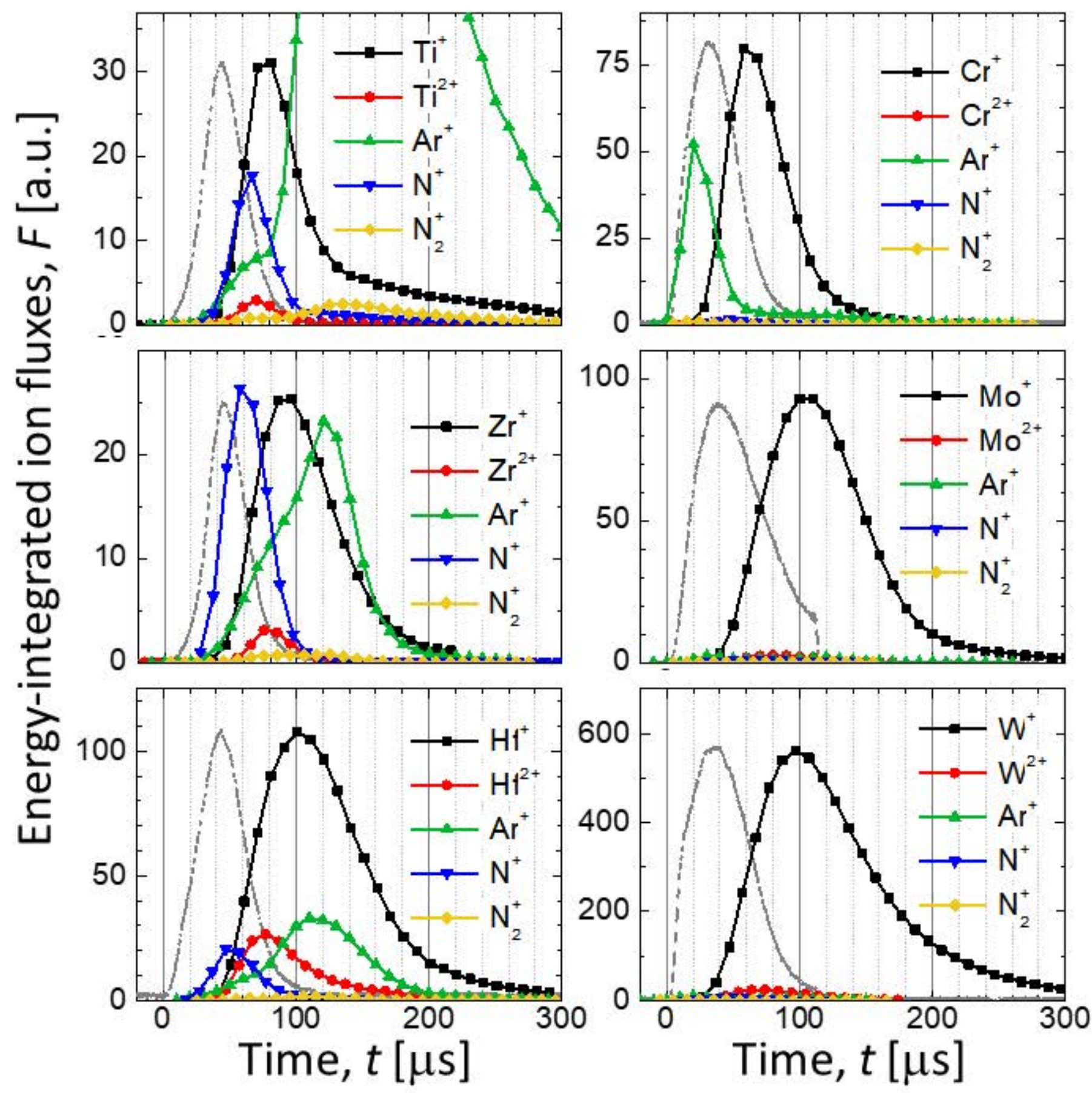


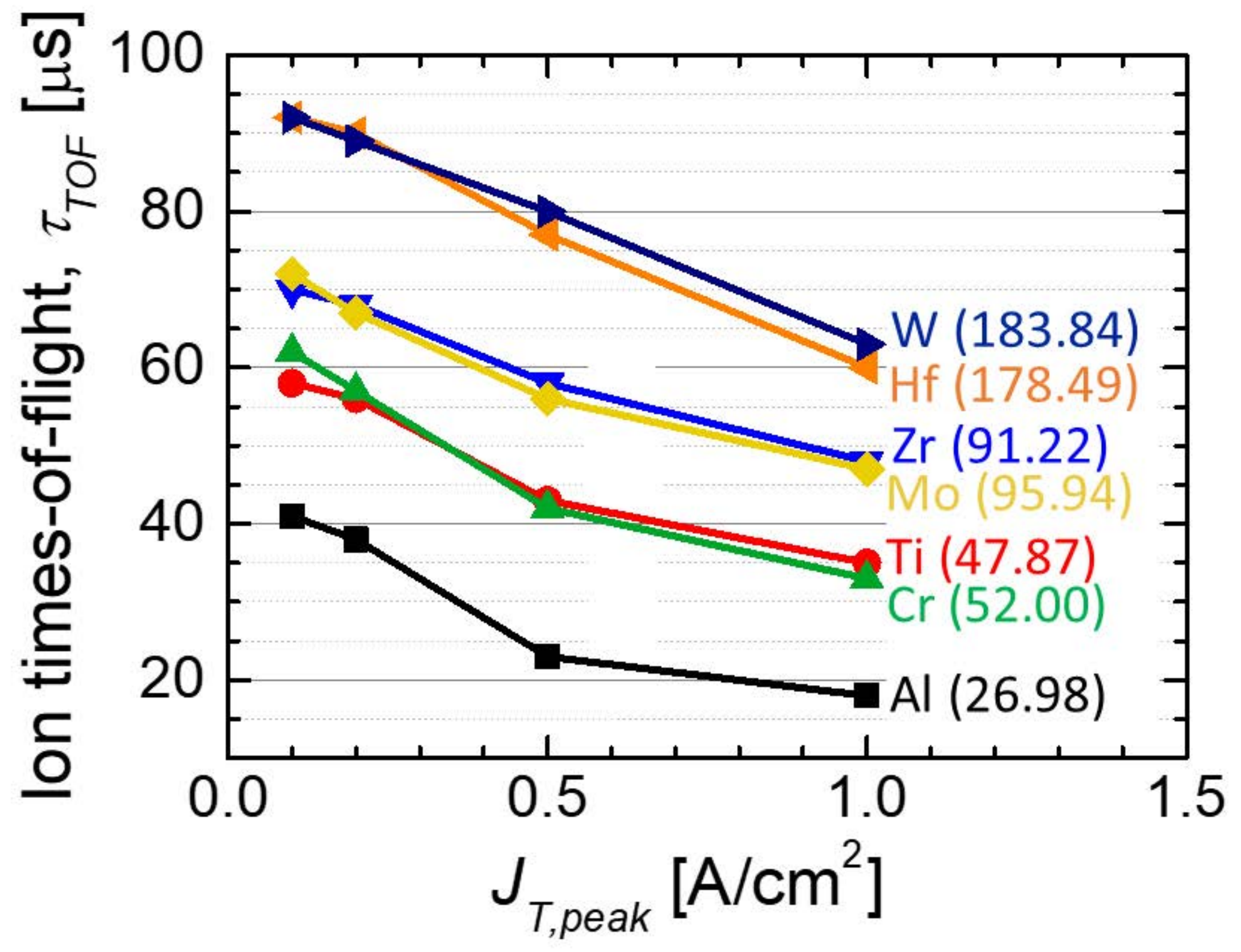






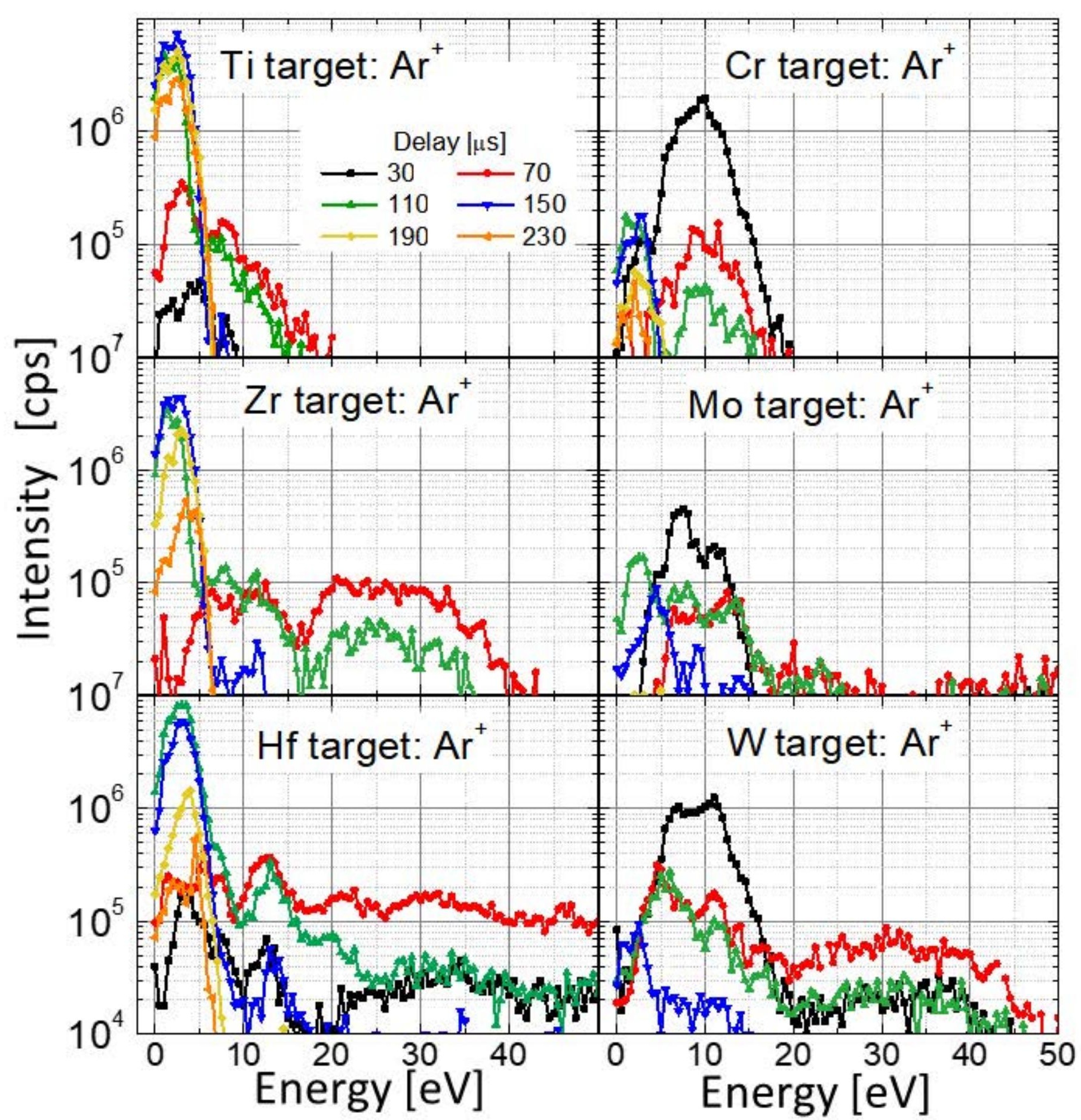




\begin{tabular}{|c|c|c|c|c|c|c|c|}
\hline TM Property & $\underset{\text { [amu] }}{m_{g}}$ & $\begin{array}{c}I P_{M e}^{1} \\
{[\mathrm{eV}]}\end{array}$ & $\begin{array}{c}I P_{M e}^{2} \\
{[\mathrm{eV}}\end{array}$ & $S_{A r \rightarrow M e}$ & $S_{M e \rightarrow M e}$ & $\begin{array}{c}\Delta H_{f}^{0} \\
\text { [eV/atom] }\end{array}$ & $\begin{array}{c}\lambda_{t h} \\
{[\mathrm{~cm}]}\end{array}$ \\
\hline $\mathrm{Ti}$ & 47.87 & 6.85 & 13.62 & $0.62(480 \mathrm{eV})$ & 0.51 & -3.4 & 9.3 \\
\hline $\mathrm{Zr}$ & 91.22 & 6.66 & 13.21 & $0.77(440 \mathrm{eV})$ & 0.51 & -3.7 & 11.2 \\
\hline $\mathrm{Hf}$ & 178.49 & 6.85 & 14.98 & $1.06(480 \mathrm{eV})$ & 0.82 & -3.7 & 15.8 \\
\hline $\mathrm{Cr}$ & 52.00 & 6.79 & 16.54 & $1.59(600 \mathrm{eV})$ & 1.34 & -1.2 & 9.6 \\
\hline Mo & 95.94 & 7.09 & 16.16 & $1.12(520 \mathrm{eV})$ & 0.72 & -0.7 & 12.2 \\
\hline $\mathbf{w}$ & 183.84 & 8.01 & 17.68 & $1.45(750 \mathrm{eV})$ & 1.02 & -0.2 & 18.1 \\
\hline
\end{tabular}

